\title{
Modified Handover Decision Algorithm in Long Term Evolution Advanced Network
}

\author{
A. S. Sada \\ Department of Electronics and \\ Telecommunications Engineering \\ Ahmadu Bello University \\ Zaria, Nigeria \\ absmsada86@gmail.com \\ A. M. S. Tekanyi \\ Department of Electronics and \\ Telecommunications Engineering \\ Ahmadu Bello University \\ Zaria, Nigeria \\ amtekanyi@abu.edu.ng
}

\author{
Z. M. Abdullahi \\ Department of Electronics and \\ Telecommunications Engineering \\ Ahmadu Bello University \\ Zaria, Nigeria \\ eagbonehime1@gmail.com \\ A. U. Mubarak \\ Centre for Wireless Communications, \\ University of Oulu P.O. Box 4500, \\ 90014, University of Oulu, Finland \\ aminu.mubarak@gmail.com
}

\author{
A. D. Usman \\ Department of Electronics and \\ Telecommunications Engineering \\ Ahmadu Bello University \\ Zaria, Nigeria \\ aliyuusman1@gmail.com \\ Y. A. Maiwada \\ Department of Electrical Engineering \\ Hassan Usman Katsina Polytechnic, \\ Katsina Nigeria \\ yusadeeq@yahoo.com
}

\begin{abstract}
Heterogeneous deployment of access points is considered as a promising technique for improved network performance in $4 \mathrm{G}$ networks. While the heterogeneity of the network results in reduced cell sizes thereby improving network coverage, inter-cell handover process becomes more complex and frequent. Therefore, the different velocities and angles of movement of the mobile users leads to excessive scanning process between neighboring cells and poses an inherent delay in the handover decision. In this paper, we present a modified handover decision algorithm whereby pedestrians and motorcyclists access the same small cell thereby reducing the scanning for different target small cells. Signal to interference plus noise ratio was considered to mitigate neighbor cell interference and introducing mobility vector prediction to accurately execute handover process. Probability of unnecessary handover and network throughput were used as metrics.
\end{abstract}

Keywords- LTE-Advanced, Handover, HetNet, SINR

\section{I." INTRODUCTION}

In its quest to increase broadband cellular networks and bring mobile users close to access points, the third generation partnership project (3GPP) developed the Long Term Evolution Networks (LTE). This next generation of wireless cellular technology supports the heterogeneous deployment of access points. Heterogeneity of the networks is the mix deployment of femtocells, picocells and microcells overlaid by a macrocell which covers larger area.

Deploying small cells in a network coverage area comes with a lot of technical, financial and environmental challenges. Technical challenges include frequent cell exchanges as a user crosses a cell boundary, financial include the cost effectiveness associated with the deployment and technical staff for the services and finally, environmental hiccups comprise of location to fix the cells and adverse effects of pollution from power generating sets to power the cell site.

Third Generation Partnership Project (3GPP) in its indefatigable effort to further enhance broadband wireless cellular networks came up with the Long Term Evolution (LTE) network system. Also termed as Fourth Generation (4G) mobile communication standard [1].Classification of base stations are of two major categories. Low-power Home evolved Node B base stations are usually installed indoors while the evolved Node B base stations are installed outdoors to improve capacity and user throughput. LTE enables lowpower base stations to connect to the Internet [2]. Handover procedure transfers active call or data session while a user is in motion from one cell to another [3].

Ref. [4] analysed an algorithm that utilizes the predictive values of Received Signal Strength (RSS) to achieve improvement in throughput and reduction in ping pong. The chosen base station was the one with the improved network throughput. Evaluation of the algorithm was based on the following: Outage Probability (OP), Ping Pong Rate (PPR), Throughput, Number of Handover (NHO) and errors associated with the prediction of the RSS.

A handover decision algorithm between femtocells was investigated by [5], using the RSS and user velocity as decision criteria. The algorithm chose a femtocell that is far away from the user equipment compared with other available adjacent femtocells. This idea suppresses unnecessary handovers before getting to the target femtocell. An Inter-femto cell handover scheme for dense femto cellular networks was proposed by [6]. Two algorithms were used to address the movement direction of the user; one for the prediction of the user mobility pattern, while the other for generating mobility rules. This paper proposes a reduced scanning process which translates to a corresponding reduction in the iteration process and reduced power dissipation of the user equipment by considering only two velocity thresholds which are low to medium speed users. Inter-cell interference was also mitigated by selecting users with high signal to interference plus noise ratio. Medium velocity threshold as classified in [7], was set and signal to interference plus noise ratio was incorporated into the mobility vector prediction technique in order to guarantee efficient handover procedure.

Organization of the remaining paper follows suite: Section II explains model. Proposed method was illustrated in Section III. And it concludes with Section IV. 


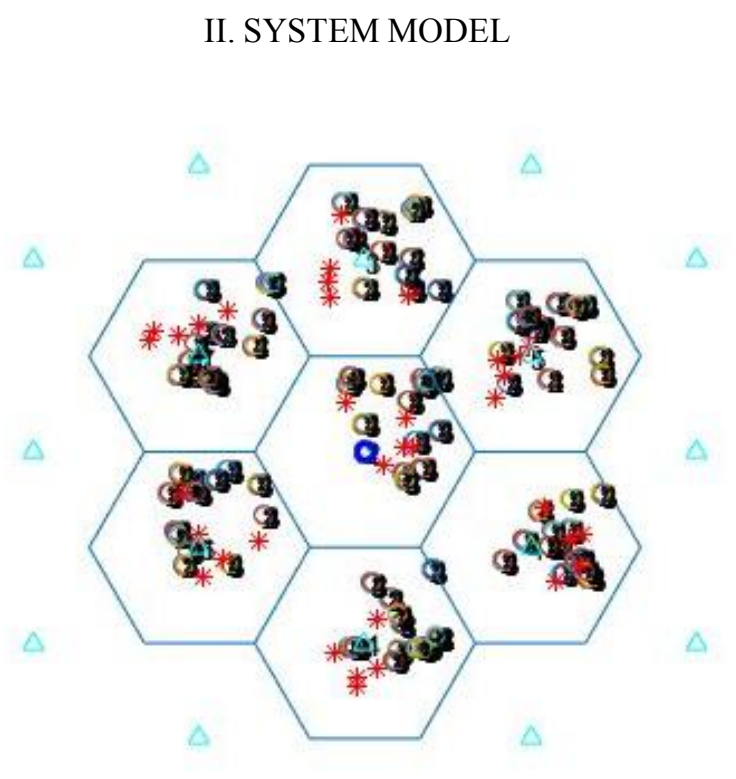

Figure 1: System Model

Consider a seven 7 hexagonal macrocells with an illustrative example depicted in 1 . The macrocell area is densely populated with 100 small cells. The blue dot is the centre point, red stars are the user equipment, the black circles are the small cells, and other small cells that are fixed on lamp posts or buildings are indicated with brown colour. Blue triangles are the macrocell areas. The small cells overlaid by the macrocell provide the users with the network coverage in the simulation environment. Low to medium velocity users are covered by same small cell in order to reduce the scanning procedure for three different velocity thresholds. This reduction in the scanning and number of iterations save battery power dissipation of the user equipment and also reduce frequent handing over between small cells as the user is moving. The user equipment adopted a random waypoint model while roaming in the coverage area.

\section{PROPOSED METHOD}

In the work of [8]; low, medium and high speed users, angles of movement in addition to signal to noise ratio were considered for a successful handover process. Significant improvements are proposed by considering only low to medium users which is aimed at reducing the number of iterations and scanning for a neighbor cell for handover. More so, for improved network throughput; signal to interference plus noise ratio was incorporated to mitigate the effect of interference from neighboring cells. Finally, a mobility vector model was introduced to ensure precise location and accurate handover is executed to the user equipment. Mobility vector prediction technique remembers the previous location of a user equipment and is able to predict the next moving direction. And it supports flexibility in movement patterns of user equipment. Figure 2 illustrates angle of $30^{\circ}$ at which the user is roaming in the coverage area which is Southwest (SW) movement to East (E).

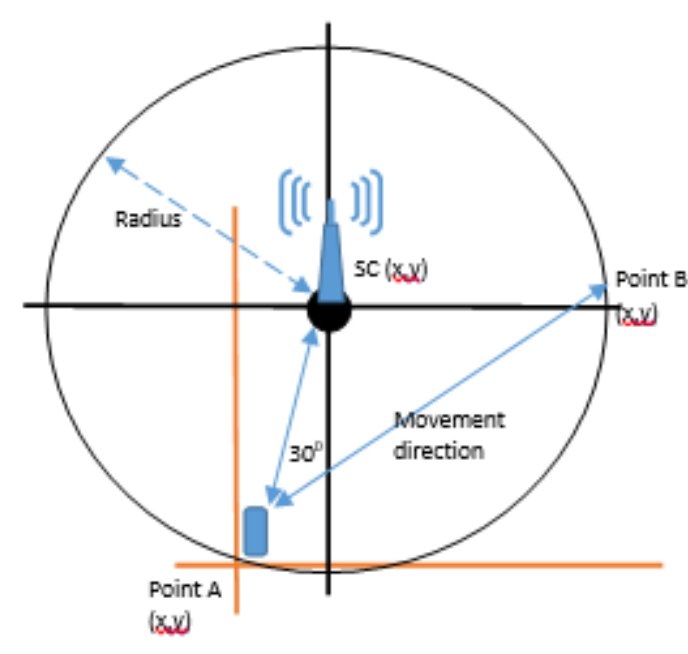

Figure 2: Angle of movement of the UE.

In 3GPP Release 11, three mobility states of users were prescribed and medium to high velocity thresholds used. For the reasons that low velocity moving users are reliably detected by the base station network and it has an ample time to complete the produces for a successful handover. Handover process is best executed before the serving cell's signal quality fades to unbearable degree. Macro-only networks come into play when medium to high velocity users could not detect reliable signal due to speed. Hence, which leads to unnecessary handover especially in massive deployment of low-power access points [9].

Mathematical models for the proposed method are explained as follows:

Set of Candidate small cells for $U E_{y}$ in one $M C$, is denoted as $S_{S C}$, and can be defined by the following equation:

$S_{s c}=\left\{\left|S C_{x} \in N_{s c}\right|\left(d_{a c t}^{w e_{y} \rightarrow s c_{x}} \leq d_{t h}\right) \wedge\left|\propto_{\text {we }}\right| \leq \alpha_{x n_{t}, t h}\right\}$

The set of SCs are contained in the shortened candidate list from which the small cell with the maximum Signal to Interference plus Noise Ratio (SINR) is selected for handover.

Furthermore, Probability of Successful Handover for the proposed modification is mathematically expressed as:

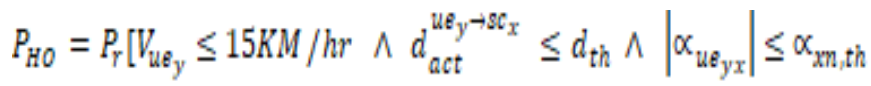

$$
\begin{aligned}
& \text { A SINR } \left.R_{\varepsilon e_{x} \rightarrow \text { we } y}^{Y}>S I N R_{m \rightarrow \text { wey }}^{Y}+M V P\right] \text {. }
\end{aligned}
$$

where

$V_{\text {ue }} \leq 15 \mathrm{KM} / \mathrm{hr}$ HO threshold velocity at base station

$V_{u e_{y}}=$ velocity of the UE

$d_{\text {act }}^{\text {ue } \rightarrow B e_{x}}=$ actual distance between the $U E$ and the SC 
$d_{t h}=$ distance threshold to form the SC list

$\left|\propto_{\text {ue }}\right|=$ angle between the $U E y$ and $S C x$

$\propto_{x n_{j}, t h}=$ angle threshold at which

\section{the SCS are included in the candidate list}

$S I N R_{a e_{x} \rightarrow \text { wey }}^{r}=$ Signal to Interference plus Noise Ratio (SINR) measured at user y from SCx

$S I N R_{m \rightarrow \text { uey }}^{Y}=$ Signal to Interference plus Noise Ratio (SINR) measured at user y from Macrocell MVP $=$ Mobility Vector Prediction

The final signal to interference plus noise ratio for the proposed method is:
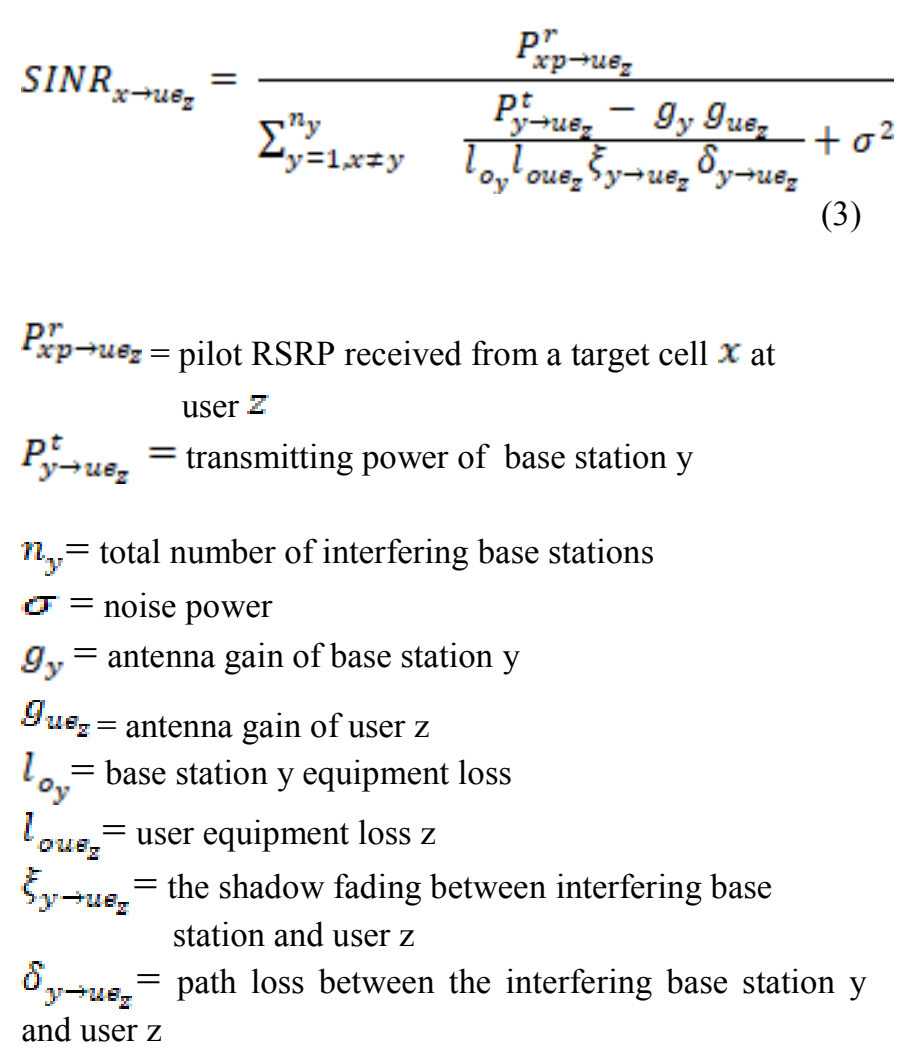

Each cell is assumed to be in a hexagonal shape to coverage holes if it is to be assumed as circular. Therefore, realistically cell borders depend on obstacles, terrains, interference and geographic area [10].

The Mobility Vector (MV) is a mobility scheme that provides a flexible mobility architecture for different motion patterns. The model supports partial changes in the mobility state of a user. It also memory dependent whereby it remembers previous state of user and predicts its next movement direction. Mobility of a user is defined by the sum of two vectors, mathematically expressed as [12]:

$$
\begin{gathered}
\vec{B}=\left(b x_{v}, b y_{v}\right) \\
\vec{V}=\left(v x_{v}, v y_{v}\right)
\end{gathered}
$$

where,

$\vec{B}$ is the base vector which defines the major direction and speed of the node and $\vec{V}$ is the Deviation vector that stores the mobility deviation from the base vector.

Therefore, the overall mobility vector from (4) and (5) is expressed as:

$$
\vec{M}=(\vec{B}+\alpha \vec{V})
$$

where $\alpha$ is an acceleration factor.

The base vector is assumed to be either the macrocell or the candidate small cells, while the deviation vector is assumed to represent the user equipment. Thus, the base vector shows the current mobility state of the macro cell or the candidate small cell and the deviation vector defines the direction and speed at which the user equipment moves towards either the candidate small cell or the macro cell.

\section{CONCLUSION}

In this paper we proposed a modified handover decision algorithm where mobility vector prediction for handover was incorporated and medium velocity threshold was set for low to medium speed users. We also proposed to mitigate the effect of inter-cell interference by considering signal to interference plus noise ratio. The metrics of probability of unnecessary handover and network throughput were used.

\section{REFERENCES}

[1] Jansen, T., Balan, I., Turk, J., Moerman, I., \& Kurner, T. (2010). Handover parameter optimization in LTE self-organizing networks. Paper presented at the Vehicular Technology Conference Fall (VTC 2010-Fall), 2010 IEEE 72nd. 1-5.

[2]" P. Bhat, S. Nagata, L. Campoy, I. Berberana, T. Derham, et. al., "LTE Advanced: an operator perspective,"IEEE Communications Magazine, vol. 50, no. 2, pp. 104-114, February 2012.

[3] Li, Y., Cao, B., \& Wang, C. (2016). Handover schemes in heterogeneous LTE networks: challenges and opportunities. IEEE Wireless Communications, 23(2), 112-117.

[4] Kalbkhani, H., Yousefi, S., \&Shayesteh, M. G. (2014). Adaptive handover algorithm in heterogeneous femtocellular networks based on received signal strength and signal-to-interference-plus-noise ratio prediction. IET Communications, 8(17), 3061-3071. doi: 10.1049/ietcom.2014.0230 
[5] Rajabizadeh, M., \&Abouei, J. (2015). An efficient femtocell-tofemtocell handover decision algorithm in LTE femtocell networks. Paper presented at the 2015 23rd Iranian Conference on Electrical Engineering (ICEE), Tehran. 213-218.

[6] Al-Shahin, F. A. (2015). Femtocell-to-Femtocell Handoff management in Dense Femtocellular networks. International Journal of Computer and Communication Engineering, 4(5), 346-353.

[7]" Haddad, Z., Mahmoud, M., Saroit, I. A., \& Taha, S. (2016, April). Secure and efficient uniform handover scheme for LTE-A networks. In Wireless Communications and Networking Conference (WCNC), 2016 IEEE (pp. 1-6). IEEE.

$[8]^{n} \quad$ F. Bai and A. Helmy, "A survey of mobility models," Wireless Adhoc Networks. University of Southern California, USA, vol. 206, 2004.

[9] Alhabo, Mohanad, L. (2017, February). Unnecessary handover minimization in two-tier heterogeneous networks. In wireless Ondemand Network Systems and Services (WONS), 2017 13th Annual Conference on, (pp. 160-164). IEEE

[10]" Zhou, Y., Lei, Z., \& Wong, S. H. (2014, May). Evaluation of Mobility Performance in 3GPP Heterogeneous Networks. In 2014 IEEE 79th Vehicular Technology Conference (VTC Spring) (pp. 1-5). IEEE.

[11]" Alhabo, M., Zhang, L., \& Nawaz, N. (2017, May). A trade-off between unnecessary handover and handover failure for heterogeneous networks. In European Wireless 2017; 23th European Wireless Conference (pp. 1-6). VDE.

[12]" Chu, X., López-Pérez, D., Yang, Y., \& Gunnarsson, F. (Eds.). (2013). Heterogeneous Cellular Networks: Theory, Simulation and Deployment. Cambridge University Press. (BOOK). 